\title{
Design of vehicle cruise control using road inclinations
}

\author{
Balázs Németh and Péter Gáspár *
}

\begin{abstract}
The paper proposes the design of velocity based on road inclinations, speed limits, a preceding vehicle on the lane and traveling time. A new control method in which the longitudinal control incorporates the brake and traction forces is also proposed to achieve the required velocity. By choosing the velocity which is appropriate according to the road and traffic information, the number of unnecessary accelerations and brakings and their durations can be significantly reduced. In the design method the modeling and robust control of the LPV theory are exploited. The operation of the longitudinal controlled system and the influences of different designed parameters are analyzed through vehicle simulations. Finally, the efficiency of the controlled system is demonstrated on a real transportation route.
\end{abstract}

\section{Introduction and motivation}

As a result of growing global requirements the automotive researchers are forced to develop flexible, reliable and economical automotive systems which require less energy during the operation. Reducing fuel consumption is an important environmental and economic requirement for vehicle systems. Since the driveline system has an important role in the emission of the vehicle, the development of the longitudinal control systems is in the focus of the research and development of the vehicle industry. The paper presents a method of how the required force and energy, thus fuel consumption can be reduced when the external road information is taken into consideration during the journey.

The controllers applied in current adaptive cruise control systems are able to take into consideration only instantaneous effects of road conditions, since they do not have information about the oncoming road sections. The cruise control systems automatically maintains a steady speed of a vehicle as set by the driver by setting the longitudinal control forces. In the paper road inclinations are taken into consideration in the design of the longitudinal control force. The aim in this calculation is to achieve a control force which is similar to the driver's requirement. For example in front of the downhill slope the driver can see the change in the curve of the road. Here the velocity of the vehicle increases, thus the control force of the vehicle before the slope can be reduced. As a result at the beginning of the slope the velocity of the vehicle decreases, thus it will increase from a lower value. Consequently, the brake system can be activated later or it may not be necessary to activate it at all. If the velocity in the next road section changes it is possible to set the adequate control force. In the knowledge of the speed limits it is also possible to save energy. Moreover, in the section of the road where a speed limit is imposed different strategies can be considered. Before the regulated section the velocity can be reduced, therefore less energy is necessary for the vehicle. Using the idea of road slope and speed limit, fuel consumption and the energy required by the actuators can be reduced. By choosing the appropriate velocity according to the road and traffic information, the number of unnecessary accelerations and brakings and their durations can be significantly reduced.

\footnotetext{
*The authors are with the Systems and Control Laboratory, Computer and Automation Research Institute, Hungarian Academy of Sciences, Kende u. 13-17, H-1111 Budapest, Hungary E-mail: [bnemeth;gaspar]@sztaki.mta.hu
} 
In the vehicle the most important longitudinal actuators are the engine, the transmission and the brake system. The engine is set at a particular revolution with corresponding consumption, torques, etc. If road conditions are known the engine can be operated more efficiently throughout the entire journey. The transmission system has effects on the engine since it creates a connection between the engine and the wheels. The selected gear affects the operation of the engine. Hence the engine and the transmission system must be handled together in a control system. Moreover, the unnecessarily frequent activation of the brake is undesirable because of the wear of the brake pad/disc and the loss in kinetic energy. The control of longitudinal dynamics requires the integration of these vehicle components, see e.g. $[8,23]$.

The method proposed in the paper takes into consideration both the inclination of the road and the speed limits. Vehicles save energy at the change of road inclinations and at the same time keep compulsory speed limits. In addition the tracking of the preceding vehicle is necessary to avoid a collision. If the preceding vehicle accelerates or decelerates the tracking vehicle must strictly track the velocity within the speed limit. Thus, this method changes the speed according to the road and traffic conditions. At the same time the efficiency of the transportation system as an important cost factor requires relatively steady speed. These requirements are in conflict and the trade-off among them can be achieved by using different weights.

Several methods in which the road conditions are taken into consideration have already been proposed, see $[7,13,14]$. The look-ahead control methods assume that information about the future disturbances to the controlled system is available. To find a compromise solution between fuel consumption and trip time leads to an optimization problem. The optimization was handled by using a receding horizon control approach in [5, 18]. In another approach the terrain and traffic flow were modeled stochastically using a Markov chain model in $[9,10]$. In $[6]$ the approach was evaluated in real experiments where the road slope was estimated by the method in [21]. Paper [3] classifies several modeling approaches for vehicle fuel consumption and emission, such as microscopic, mesoscopic and macroscopic modeling methods. From the aspect of microscopic approach, models of vehicle dynamics are preferred in the paper. Alternative truck lane management strategies are evaluated in [19]. The efficiency of this method is presented by different scenarios, which show that using these methods travel time, energy and the emission of the vehicle can be reduced. $[19,20]$ present modeling methods for the design of route guidance strategies and the reliable estimation of travel time. The preliminary results of the research are also published in $[14,15]$.

The aim of the design method is to calculate the longitudinal forces by using an optimization method. The optimal solution is built into a closed-loop interconnection structure in which a robust controller is designed by using a Linear Parameter Varying (LPV) method. In the LPV method uncertainties, disturbances and nonlinear properties of the system are also handled. The optimization task uses general and scalable vehicle parameters which are known, measured or can be estimated. The real physical inputs of the system (throttle, gear position, brake pressure) are calculated by using the longitudinal force required by velocity tracking. The specific components such as actuators occur in the implementation task. An important feature of the method is that the optimization task and the implementation task are handled separately. Consequently, the method can be implemented in an ECU (electronic control unit) in practice.

This paper is organized as follows: Section 2 formalizes the road inclinations and speed limits in a control-oriented model and establishes the concept of weighting strategy. Section 3 presents the optimization of vehicle cruise control by the appropriate choice of the weights. Section 4 develops the control scheme of velocity tracking according to the optimal weights and performs the robust control design, while Section 5 proposes the architecture of the control system. Section 6 shows the operation of the controlled system and analyzes the effects of vehicle and control parameters. Finally, Section 7 summarizes the conclusion remarks. 


\section{Consideration of road conditions}

In this section the road inclinations and speed limits are formalized in a control-oriented model. First the road ahead of the vehicle is divided into several sections and reference velocities are selected for them. The rates of the inclinations of the road and those of the speed limits are assumed to be known at the endpoints of each section. Second the road sections are qualified by different weights, which have an important role in control design. The appropriate selection of the weights creates a balance between the velocity of the vehicle and the effects of road conditions. The knowledge of the road inclinations is a necessary assumption for the calculation of the velocity signal. In practice the slope of the road can be obtained in two ways: either a contour map which contains the level lines is used, or an estimation method is applied. In the former case a map used in other navigation tasks can be extended with slope information. Several methods have been proposed for slope estimation. They use cameras, laser/inertial profilometers, differential GPS or a GPS/INS systems, see $[1,11,4]$. An estimation method based on a vehicle model and Kalman filters was proposed by [12]. The detection of a speed limit sign is usually based on a video camera.

The principle of the consideration of road conditions is the following. It is assumed that the vehicle travels in a segment from the initial point (beginning of the road section) to the first division point. The velocity at the initial point is predefined and it is called original velocity. The journey is carried out with constant longitudinal force. The dynamics of the vehicle is described between the initial and the first division points. An important question is how velocity should be selected at the initial point (called modified velocity) at which the reference velocity of the first point can be reached using a constant longitudinal force. The thought can be extended to the next segments and division points. In case of $n$ number of segments $n$ equations are formalized between the first and the end points.

The number of the segments is important. For example in the case of flat roads it is enough to use relatively few section points because the slopes of the sections do not change abruptly. In the case of undulating roads it is necessary to use relatively large number of section points and shorter sections, because it is assumed in the algorithm that the acceleration of the vehicle is constant between the section points. Thus, the road ahead of the vehicle is divided unevenly, which is consistent with the topography of the road.

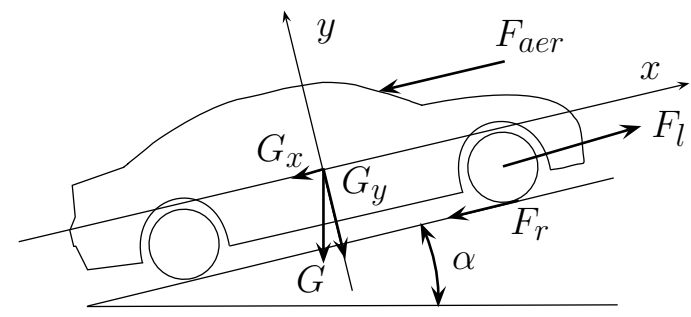

Figure 1: Simplified vehicle model

The simplified model of the longitudinal dynamics of the vehicle is shown in Figure 1. The longitudinal movement of the vehicle is influenced by the traction force $F_{l}$ as the control signal and disturbances $F_{d}$. Several longitudinal disturbances influence the movement of the vehicle. The rolling resistance is modeled by an empiric form $F_{r}=F_{z} f_{0}\left(1+\kappa \dot{\xi}^{2}\right)$, where $F_{z}$ is the vertical load of the wheel, $f_{0}$ and $\kappa$ are empirical parameters depending on tyre and road conditions and $\dot{\xi}$ is the velocity of the vehicle, see [16]. The aerodynamic force is formulated as $F_{a e r}=0.5 C_{w} \rho A_{0} \dot{\xi}_{r e l}^{2}$, where $C_{w}$ is the drag coefficient, $\rho$ is the density of air, $A_{0}$ is the reference area, $\dot{\xi}_{r e l}$ is the velocity of vehicle relative to the air. In case of a lull $\dot{\xi}_{\text {rel }}=\dot{\xi}$, which is assumed in the paper. The longitudinal component of the weighting force is $G_{x}=m g \sin \alpha$, where $\mathrm{m}$ is the mass of the vehicle and $\alpha$ is the angle of the slope. The acceleration of the vehicle is the following: $\ddot{\xi}=\left(F_{l}-F_{d}\right) / m$, where $m$ is the mass of the 
vehicle, $\xi$ is the position of the vehicle, and $F_{l}, F_{d}$ are the traction force and the disturbance force $\left(F_{d}=F_{r}+F_{a e r}+G_{x}\right)$, respectively.

The predicted course of the vehicle can be divided into sections using $n+1$ number of points as Figure 2 shown. Although between the points may be acceleration and declaration an average speed is used. Thus, the rate of accelerations of the vehicle is considered to be constant between these points. In this case the movement of the vehicle using simple kinematic equations is: $s_{1}=\dot{\xi}_{0}\left(\dot{\xi}_{1}-\dot{\xi}_{0}\right) / \ddot{\xi}+\left(\dot{\xi}_{1}-\dot{\xi}_{0}\right)^{2} / 2 / \ddot{\xi}$, where $\dot{\xi}_{0}$ is the velocity of vehicle at the initial point, $\dot{\xi}_{1}$ is the velocity of vehicle at the first point and $s_{1}$ is the distance between these points.

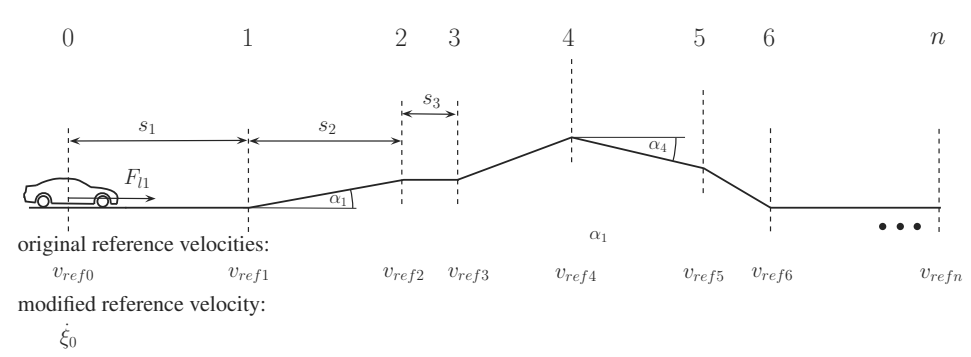

Figure 2: Division of road

Thus the velocity of the first section point is the following:

$$
\dot{\xi}_{1}^{2}=\dot{\xi}_{0}^{2}+2 \ddot{\xi}_{1}=\dot{\xi}_{0}^{2}+\frac{2}{m} s_{1}\left(F_{l 1}-F_{d 1}\right)
$$

The velocity of the first section point $\dot{\xi}_{1}^{2}$ is defined as the reference velocity $v_{r e f, 1}^{2}=\dot{\xi}_{1}^{2}$. This relationship also applies to the next road section: $\dot{\xi}_{2}^{2}=\dot{\xi}_{1}^{2}+2 \ddot{\xi}_{2}$.

The purpose of considering road conditions is to determine a control force by which the vehicle can travel along its way. It is important to emphasize that the longitudinal force $F_{l 1}$ is known only the first section. Moreover, the longitudinal forces $F_{l i}, i>1$ are not known during the traveling in the first section. Therefore at the calculation of the control force it is assumed that additional longitudinal forces will not act on the vehicle, i.e., the longitudinal forces $F_{l i}, i>1$ will not affect the next sections. At the same time the disturbances from road slope are known ahead. Consequently, the velocity of the second section point $\dot{\xi}_{2}^{2}$, which is defined as the reference velocity $v_{r e f, 2}^{2}=\dot{\xi}_{2}^{2}$, is as follows:

$$
\dot{\xi}_{2}^{2}=\dot{\xi}_{0}^{2}+\frac{2}{m}\left(s_{1} F_{l 1}-s_{1} F_{d 1}-s_{2} F_{d 2}\right) .
$$

Similarly, the velocity of the vehicle can be formalized in the next $n$ section points. Using this principle a velocity-chain, which contains the required velocities along the way of the vehicle, is constructed. At the calculation of the control force it is assumed that additional longitudinal forces $F_{l i}, i \in[2, n]$ will not affect the next sections. The velocities of vehicle are described at each section point of the road by using similar expressions to (1). The velocity of the $n^{\text {th }}$ section point is the following:

$$
\dot{\xi}_{n}^{2}=\dot{\xi}_{0}^{2}+\frac{2}{m}\left(s_{1} F_{l 1}-\sum_{i=1}^{n} s_{i} F_{d i}\right)=v_{r e f, n}^{2}
$$

It is also an important goal to track the momentary value of the velocity. It can also be considered in the following equation:

$$
\dot{\xi}_{0}^{2} \rightarrow v_{r e f, 0}^{2} .
$$


The $F_{d i}$ disturbance force can be divided in two parts: the first part is the force resistance from road slope $F_{d i, r}$, while the second part $F_{d i, o}$ contains all of the other resistances such as rolling resistance, aerodynamic forces etc. We assume that $F_{d i, r}$ is known while $F_{d i, o}$ is unknown. $F_{d i, r}=G_{x}=m g \sin \alpha_{i}$ depends on the mass of the vehicle and the angle of the slope $\alpha_{i}$. When the control force $F_{l 1}$ is calculated, only $F_{d 1, o}$ influences the vehicle of all of the unmeasured disturbances. In the control design the effects of the unmeasured disturbances $F_{d i, o}, i \in\{2, n\}$ are ignored. The consequence of this assumption is that the model does not contain all the information about the road disturbances, therefore it is necessary to design a robust speed controller. This controller can ignore the undesirable effects. Consequently, the equations of the vehicle at the section points are calculated in the following way:

$$
\begin{aligned}
\dot{\xi}_{0}^{2}=v_{r e f, 0}^{2} & \\
\dot{\xi}_{0}^{2}+\frac{2}{m} s_{1} F_{l 1}-\frac{2}{m} s_{1} F_{d 1, o} & =v_{r e f, 1}^{2}+\frac{2}{m} s_{1} F_{d 1, r} \\
\dot{\xi}_{0}^{2}+\frac{2}{m} s_{1} F_{l 1}-\frac{2}{m} s_{1} F_{d 1, o} & =v_{r e f, 2}^{2}+\frac{2}{m}\left(s_{1} F_{d 1, r}+s_{2} F_{d 2, r}\right) \\
\vdots & \\
\dot{\xi}_{0}^{2}+\frac{2}{m} s_{1} F_{l 1}-\frac{2}{m} s_{1} F_{d 1, o} & =v_{r e f, n}^{2}+\frac{2}{m} \sum_{i=1}^{n} s_{i} F_{d i, r}
\end{aligned}
$$

The vehicle travels in traffic and it may happen that the vehicle is overtaken. Because of the risk of collision it is necessary to consider the preceding velocity on the lane:

$$
\dot{\xi}_{0}^{2} \rightarrow v_{\text {lead }}^{2}
$$

instead of the basic equation (4).

In the next step weights $\gamma_{1}, \gamma_{2}, \ldots, \gamma_{n}$ are applied to both the reference velocity and the road slope in equation (5). An additional weight $Q$ is applied in equation (4). An additional weight $W$ is applied in (6). $W$ represents the tracking of the velocity of the preceding vehicle $v_{\text {lead }}$ in order to avoid a collision. The weights should sum up to one, i.e. $\gamma_{1}+\gamma_{2}+\ldots+\gamma_{n}+Q+W=1$. While the weights $\gamma_{i}$ represent the rate of the road conditions, weight $Q$ has an essential role: it determines the tracking requirement of the current reference velocity $v_{\text {ref, } 0}$. By increasing $Q$ the momentary velocity becomes more important while road conditions become less important. Similarly, by increasing $W$ the road conditions and the momentary velocity become negligible. By summarizing equation (5) and taking the weights into consideration the following formula is yielded:

$$
\dot{\xi}_{0}^{2}+\frac{2}{m} s_{1}(1-Q-W) F_{l 1}-\frac{2}{m} s_{1}(1-Q-W) F_{d 1, o}=\vartheta
$$

where the value $\vartheta$ depends on the road slopes, the reference velocities and the weights

$$
\vartheta=W v_{\text {lead }}^{2}+Q v_{\text {ref }, 0}^{2}+\sum_{i=1}^{n} \gamma_{i} v_{r e f, i}^{2}+\frac{2}{m} \sum_{i=1}^{n} s_{i} F_{d i, r} \sum_{j=i}^{n} \gamma_{j} .
$$

In order to take the road conditions into consideration in the control design (7) is applied as a performance of the controlled system. Note that weights have an important role in control design. By making an appropriate selection of the weights the importance of the road condition is taken into consideration. For example when $Q=1$ and $W=\gamma_{i}=$ $0, i \in[1, n]$ the control exercise is simplified to a cruise control problem without any road conditions. When equivalent weights are used the road conditions are considered with the same importance, i.e., $Q=\gamma_{1}=\gamma_{2}=\ldots=\gamma_{n}$ and $W=0$. When $W=1$ and $Q=$ $\gamma_{i}=0, i \in[1, n]$ only the tracking of the preceding vehicle is carried out. The optimal 
determination of the weights has an important role, i.e., to achieve a balance between the current velocity and the effect of the road slope. Consequently, a balance between the velocity and the economy parameters of the vehicle is formalized.

In addition to the consideration of road conditions it is also important to consider the traffic environment. It means that the preceding vehicle must be considered in the reference velocity design because of the risk of collision. Thus, all of the kinetic energy of the vehicle is dissipated by friction. This estimation of the safe stopping distance may be conservative in a normal traffic situation, where the preceding vehicle also brakes, therefore the distance between the vehicles may be reduced. In this paper the safe stopping distance between the vehicles is determined according to the 91/422/EEC, 71/320/EEC UN and EU directives (in case of $M_{1}$ vehicle category, the velocity in $\left.k m / h\right): d_{s t}=0.1 \dot{\xi}_{0}+\dot{\xi}_{0}^{2} / 150$. It is also necessary to consider that without the preceding vehicle the consideration of safe stopping distance is neither possible nor necessary. The consideration of the preceding vehicle is determined by $W$, therefore this weight is determined according to Figure 3.

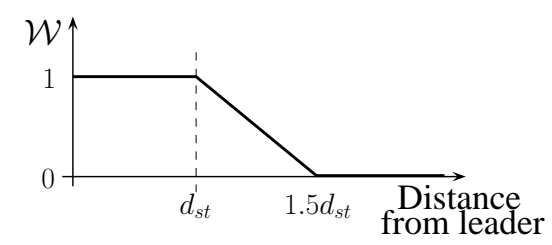

Figure 3: Selection of weight W

In the final step a control-oriented vehicle model, in which reference velocities and weights are taken into consideration, is constructed. The momentary acceleration of the vehicle is expressed in the following way: $\ddot{\xi}_{0}=\left(F_{l}-F_{d, o}-F_{d 1, r}\right) / m$ where $F_{d 1, r}=m g \sin \alpha$. Equation (7) is rearranged:

$$
\dot{\xi}_{0}=\lambda
$$

where the parameter $\lambda$ is calculated in the following way based on the designed $\vartheta$ :

$$
\lambda=\sqrt{\vartheta-2 s_{1}(1-Q-W)\left(\ddot{\xi}_{0}+g \sin \alpha\right)}
$$

Consequently, the road conditions can be considered by velocity tracking. The momentary velocity of vehicle $\dot{\xi}$ should be equal to parameter $\lambda$, which contains the road information. The calculation of $\lambda$ requires the measurement of the longitudinal acceleration $\ddot{\xi}_{0}$.

\section{Optimization of the vehicle cruise control}

Equation (7) shows that the modified velocity $\dot{\xi}_{0}$ depends on the weights $\left(Q, W\right.$ and $\left.\gamma_{i}\right)$. By choosing these values the effects of road conditions can be tuned. In this section the task is to find an optimal selection of the weights in such a way that both the minimization of control force and the traveling time are taken into consideration.

The unmeasured disturbances such as rolling resistance and aerodynamic force can be expressed by the quadratic form of the velocity. The sum of these forces can be written in the following form:

$$
F_{d 1, o}=F_{r}+F_{a e r}=F_{z} f_{0}\left(1+\kappa \dot{\xi}_{0}^{2}\right)+0.5 C_{w} \rho A_{0} \dot{\xi}_{0}^{2}=A+T \dot{\xi}_{0}^{2}
$$

where $A$ and $T$ are assumed to have been calculated in advance. By substituting the expression (11) in (7) the following equation is obtained:

$$
\dot{\xi}_{0}^{2}+\frac{2 s_{1}}{m}(1-Q-W) F_{l 1}-\frac{2 s_{1}}{m}(1-Q-W)\left(A+T \dot{\xi}_{0}^{2}\right)=\vartheta
$$


Equation (12) shows that $F_{l 1}$ depends only on the weights in the following way:

$$
F_{l 1}=\beta_{0}(Q)+\beta_{1}(Q) \gamma_{1}+\beta_{2}(Q) \gamma_{2}+\ldots+\beta_{n}(Q) \gamma_{n}
$$

Since $\beta_{i}$ depends on the weight $Q$, therefore $F_{l 1}$ depends on the weights $Q$ and $\gamma_{i}$.

The vehicle cruise control problem can be divided into two optimization problems in the following forms:

Optimization 1: The longitudinal control force must be minimized, i.e., $\left|F_{l 1}\right| \rightarrow$ Min!. Instead, in practice the $F_{l 1}^{2} \rightarrow$ Min! optimization is used because of the simpler numerical computation.

Optimization 2: The difference between momentary velocity and modified velocity must be minimized, i.e., $\left|v_{r e f, 0}-\dot{\xi}_{0}\right| \rightarrow$ Min!

The two optimization criteria lead to different optimal solutions. In the first criterion the road inclinations and speed limits are taken into consideration by using appropriately chosen weights $\bar{Q}, \bar{\gamma}_{i}$. At the same time the second criterion is optimal if the information is ignored. In the latter case the weights are noted by $\breve{Q}, \breve{\gamma}_{i}$.

The first performance (Optimization 1) is met by the transformation of the quadratic form into the linear programming using the simplex algorithm. It leads to the following form:

$$
\bar{F}_{l 1}^{2}\left(\bar{Q}, \bar{\gamma}_{i}\right)=\left(\beta_{0}(\bar{Q})+\beta_{1}(\bar{Q}) \bar{\gamma}_{1}+\beta_{2}(\bar{Q}) \bar{\gamma}_{2}+\ldots+\beta_{n}(\bar{Q}) \bar{\gamma}_{n}\right)^{2}
$$

with the following constrains

$$
\begin{array}{r}
0 \leq \bar{Q}, \bar{\gamma}_{i} \leq 1 \\
\bar{Q}+\sum \bar{\gamma}_{i}=1-W
\end{array}
$$

This task is nonlinear because of the weights. The optimization task is solved by a linear programming method, such as the simplex algorithm.

The second performance criterion (Optimization 2) is also taken into consideration. The optimal solution can be determined in a relatively easy way since the vehicle tracks the predefined velocity if the road conditions are not considered. Consequently, the optimal solution is achieved by selecting the weights in the following way: $\breve{Q}=1$ and $\breve{\gamma}_{i}=0, i \in[1, n]$.

A balance between the two performances must be achieved, which is based on a tuning of the weights. The first performance is met by selecting the weights $\bar{Q}, \bar{\gamma}_{i}$ and minimizing (13). The second performance is met by selecting constant weights $\breve{Q}=1-W, \breve{\gamma}_{i}=0$. Several methods can be applied in this task. In the proposed method two further performance weights, i.e., $R_{1}$ and $R_{2}$, are introduced. The performance weight $R_{1}\left(0 \leq R_{1} \leq 1\right)$ is related to the importance of the minimization of the longitudinal control force $F_{l 1}$ (Optimization 1) while the performance weight $R_{2}\left(0 \leq R_{2} \leq 1\right)$ is related to the minimization of $\left|v_{r e f, 0}-\dot{\xi}_{0}\right|$ (Optimization 2). There is a constraint according to the performance weights $R_{1}+R_{2}=1$. Thus the performance weights, which guarantee balance between optimizations tasks, are calculated in the following expressions:

$$
\begin{aligned}
Q & =R_{1} \bar{Q}+R_{2} \breve{Q}=R_{1} \bar{Q}+R_{2} \\
\gamma_{1} & =R_{1} \bar{\gamma}_{1}+R_{2} \breve{\gamma}_{1}=R_{1} \bar{\gamma}_{1} \\
\vdots & \\
\gamma_{n} & =R_{1} \bar{\gamma}_{n}+R_{2} \breve{\gamma}_{n}=R_{1} \bar{\gamma}_{n}
\end{aligned}
$$

Based on the calculated performance weights the modified velocity can be determined by using (7). 
The tracking of the preceding vehicle is necessary to avoid a collision, therefore $W$ is not reduced. If the preceding vehicle accelerates, the tracking vehicle must accelerate as well. As the velocity increases so does the braking distance, therefore the following vehicle strictly tracks the velocity of the preceding vehicle. On the other hand it is necessary to prevent the velocity of the vehicle from increasing above the official speed limit. Therefore the tracked velocity of the preceding vehicle is limited by the maximum speed. If the preceding vehicle accelerates and exceeds the speed limit the following vehicle may fall behind.

Finally, it is necessary to analyze how the weights $Q$ and $\gamma_{i}$ modify the optimal solution of the first performance $\left(\bar{Q}\right.$ and $\left.\bar{\gamma}_{i}\right)$, i.e., the weights based on the road inclinations and speed limits. If (16) is substituted in (13) the coefficients $\beta_{i}, i \in[0, n]$ are calculated by using algebraic relationships.

$$
\begin{aligned}
\beta_{0}(Q) & =\frac{m}{2 s(1-Q-W)}\left\{Q v_{r e f, 0}^{2}+(1-Q-W) \frac{2 s}{m} A+\dot{\xi}_{0}^{2}\left[(1-Q-W) \frac{2 s}{m} T-1\right]\right\}= \\
& =\frac{m\left(\bar{Q} v_{r e f, 0}^{2}-\dot{\xi}_{0}^{2}\right)}{2 s R_{1}(1-\bar{Q}-\bar{W})}+A+T \dot{\xi}_{0}^{2}+\frac{m v_{r e f, 0}^{2}\left(1-R_{1}\right)}{2 s R_{1}} \\
& =\beta_{0}(\bar{Q})+\frac{m v_{r e f, 0}^{2}\left(1-R_{1}\right)}{2 s R_{1}} \\
\beta_{i}(Q) \gamma_{i} & =\gamma_{i} \frac{m}{2 s(1-Q-W)}\left(v_{r e f, j}^{2}+\frac{2 s}{m}\left(F_{d 1_{1}}+\ldots+F_{d i_{1}}\right)\right)= \\
& =\bar{\gamma}_{j} \frac{m}{2 s(1-\bar{Q}-\bar{W})}\left(v_{r e f, j}^{2}+\frac{2 s}{m}\left(F_{d 1_{1}}+\ldots+F_{d i_{1}}\right)\right)= \\
& =\beta_{i}(\bar{Q}) \bar{\gamma}_{i}
\end{aligned}
$$

The expressions show that the weight $\bar{Q}$ changes because $\beta_{0}(Q) \neq \beta_{0}(\bar{Q})$. At the same time the other optimal weights $\bar{\gamma}_{i}$ do not change at a fix weight $\bar{Q}$. Consequently, the common weights $\left(Q, \gamma_{i}\right)$ are not the optimum of the first performance. If $R_{1}=1$, which means that only energy minimization is considered, the optimal weights and the minimization function are the same. Therefore it is demonstrated that the two performances (and the two optimization criteria) are in conflict, they do not have a common optimum.

The optimization task is solved only on a bounded range of the weights: $0 \leq Q, \gamma_{i} \leq 1$ and $Q+W+\sum \gamma_{i}=1$. The solution of this task is difficult and it requires a great deal of computation besides decreasing the condition number of $\Phi$. In practice the numerical computations result in optimal weights, which change very sharply as a jump signal. In order to avoid this phenomenon the weights are filtered by low-pass filters to obtain smooth signals.

\section{LPV control design method}

The velocity tracking requires a controller which generates the longitudinal force. In this section a high-level controller which calculates the longitudinal force is required. Note that the realization of the longitudinal force requires another low-level controller which sets the throttle angle and the gear position in the case of driving, or brake pressure in the case of braking. The longitudinal dynamics of the vehicle is formalized in the following form: $m \ddot{\xi}_{0}=F_{l 1}-F_{d 1}$. Both the driveline and braking systems have delays in their operations. The delay is caused by different factors such as the inertia of the driveline, the burning processes and injection, the turbo lag at driving, and the inertia of the hydraulic (or pneumatic) component in the braking system. The actuator dynamics is approximated by a first-order system [22]:

$$
\dot{F}_{l 1}=\frac{1}{\tau}\left(\tilde{F}_{l 1}-F_{l 1}\right)
$$


where $F_{l 1}$ is the realized force, $\tilde{F}_{l 1}$ is the desired force of the vehicle, and $\tau$ is the delay of the system. Moreover, the delay parameter differs at driving $\left(\tau_{d}\right)$ and at braking $\left(\tau_{b}\right)$. More precisely, at braking the delay is less than at driving, i.e., $\tau_{d}>\tau_{b}$. Therefore the delay parameter is a varying component in the system.

The equations of the longitudinal dynamics and actuator dynamics are transformed into the following state-space representation form:

$$
\dot{x}=A x+B_{1} F_{d 1}+B_{2} \tilde{F}_{l 1}
$$

where the state vector is $x=\left[\begin{array}{c}\dot{\xi}_{0} \\ F_{l 1}\end{array}\right]$ and the matrices in the state space representation are $A=\left[\begin{array}{ll}0 & 1 / m \\ 0 & -1 / \tau\end{array}\right], B_{1}=\left[\begin{array}{c}-1 / m \\ 0\end{array}\right], B_{2}=\left[\begin{array}{c}0 \\ 1 / \tau\end{array}\right]$. In the state space description the operations of the driving and the braking are handled simultaneously. Since $\tau_{d}>\tau_{b}$ the model is able to separate the driving and braking cases depending on $\tau$.

The LPV model is based on the possibility of rewriting the plant in a form in which time varying terms can be hidden with suitably-defined scheduling variables. The LPV modeling approaches allow us to take into consideration the time varying effects in the state space description. Furthermore this state space representation of the LPV model is valid in the entire operating region of interest. The advantage of LPV methods is that the controller meets robust stability and performance demands in the entire operational interval, since the controller is able to adapt to the current operational conditions.

Selecting the scheduling variable $\tau$ the model can be transformed into an LPV model:

$$
\dot{x}=A(\rho) x+B_{1} F_{d 1}+B_{2}(\rho) \tilde{F}_{l 1}
$$

where $\rho$ is the scheduling variable:

$$
\rho=\left\{\begin{array}{cc}
\tau_{d} & \text { in driving case } \\
\tau_{b} & \text { in braking case }
\end{array}\right.
$$

Equation (9) leads to a tracking problem. The aim of tracking is to ensure that the system output follows a reference value with an acceptable error, which is the performance of the system. The explicit mathematical description of the optimization problem is as follows:

$$
\left(\dot{\xi}_{0}-\lambda\right) \longrightarrow M i n !
$$

where parameter $\lambda$ is the reference value. In the velocity tracking problem, $z=\dot{\xi}_{0}-\lambda$ is the performance output. The closed-loop interconnection structure, which includes the feedback structure of the model $P$ and controller $K$, is shown in Figure 4.

The control design is based on a weighting strategy. The purpose of weighting function $W_{p}$ is to define the performance specifications of the control system, i.e., the velocity of the vehicle must ensure the tracking of the reference signal with an acceptable error. They can be considered as penalty functions, i.e., the weights should be large where small signals are desired and small where large performance outputs can be tolerated. The formalized vehicle model approximates the driveline/braking system with a rigid body model. In case of real vehicles both driveline and braking systems have torsional or longitudinal vibrations. The natural frequencies of these effects increase on higher frequencies. The weighting function $W_{p}$ is selected as $W_{p}=\alpha /(T s+1)$, where $\alpha$ and $T$ are constants.

The purpose of the weighting function $W_{n}$ is to reflect the sensor noise, while $W_{w}$ represents the effect of longitudinal disturbances. In the modeling an unstructured uncertainty is modelled by connecting an unknown but bounded perturbation block $\left(\|\Delta\|_{\infty}<1\right)$ to the plant. The unstructured perturbation is connected to the plant in an output multiplicative structure. The magnitude of multiplicative uncertainty is handled by a weighting function 


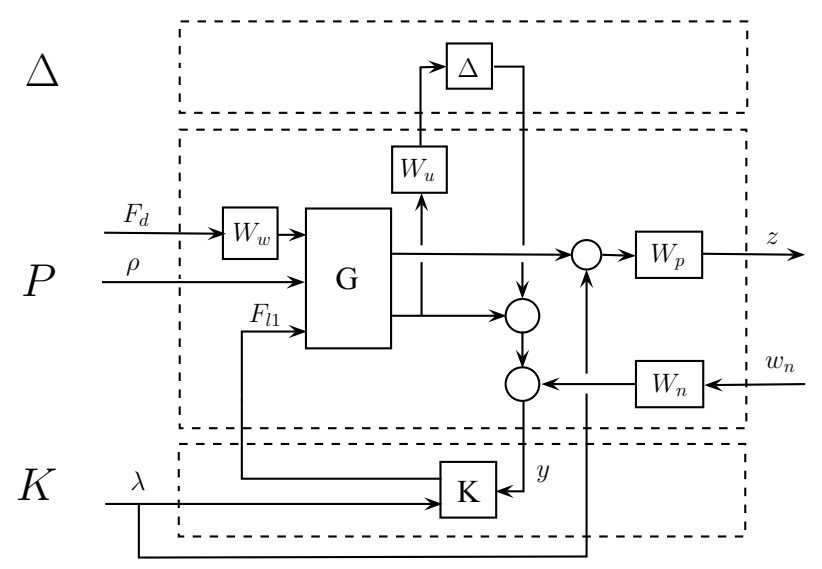

Figure 4: Closed-loop interconnection structure

$W_{u}$. The weighting functions $W_{u}, W_{w}$ and $W_{n}$ are selected in linear and proportional forms. Note that although weighting functions are formalized in the frequency domain, their statespace representation forms are applied in the weighting strategy and in the control design.

In the design of the control system the quadratic LPV performance problem is to choose the parameter-varying controller in such a way that the resulting closed-loop system is quadratically stable and, with zero initial conditions, the induced $\mathcal{L}_{2}$ norm from $w$ to $z$ is less than $\gamma$.

$$
\|M(\rho)\|_{\infty}=\inf _{K} \sup _{\rho \in \mathcal{F}_{\mathcal{P}}\|w\|_{2} \neq 0, w \in \mathcal{L}_{2}} \sup _{\|w\|_{2}} \frac{\|z\|_{2}}{\| w}
$$

The existence of a controller that solves the quadratic LPV $\gamma$-performance problem can be expressed as the feasibility of a set of Linear Matrix Inequalities (LMIs), which can be solved numerically.

The LPV systems in early applications were based on a single Lyapunov function (SLF) approach, in which the variation of the scheduling variables can be arbitrarily fast. This work has been extended to analysis and synthesis by incorporating a parameter dependent Lyapunov function, see $[2,17,24]$. The incorporation of a parameter dependent Lyapunov function implies a potentially less conservative approach by addressing limitations on the rate of change of the parameters $\rho$. However, the control design leads to infinite dimensional convex feasibility conditions. These conditions can, in general, only be obtained approximately, by selecting grid points from the whole set, thus it is converted into finite dimensional LMIs.

Note that if parameter-dependent Lyapunov functions are used, the controller designed depends explicitly on $\dot{\rho}$. Thus, in order to construct a parameter-dependent controller, both $\rho$ and $\dot{\rho}$ must be measured or available. When $\dot{\rho}$ is not measured in practice, a suitable extrapolation algorithm must be used to achieve an estimation of the parameter $\dot{\rho}$. The disadvantage of this approach is that the sources of the scheduling variables are not independent. Balas et al. proposed a possible method to perform a $\rho$-dependent change of variables to remove $\dot{\rho}$ dependence, see [17].

When the LPV controller has been synthesized, the relation between the state, or output, and the parameter $\rho=\sigma(x)$ is used in the LPV controller, such that a nonlinear controller is obtained. Note that it is assumed that $\sigma(x)$ is measured or depends only on measured signals. According to the properties of the LPV description the LPV system with $\rho=\sigma(x)$ is equal to the nonlinear model. The realization of an LPV controller poses a problem, which must be handled. The control design is performed in continuous-time, in which it is assumed that the scheduling variable is known in continuous-time. However, $\rho$ is measured 
only at sampling times. Instead of getting a fixed dependence of system matrices on $\rho$, the matrices are only known at a discrete $\rho$ values. The suitable sampling time must be selected according to the physical system; however, the real sampling time is modified by the implementation possibilities. Thus the determination of the parameters during the intervals between sampling times is a difficult theoretical problem. A simple procedure applied in practice uses a zero-order hold method between sampling times. A better solution of the approximation is based on polynomial or rational functions through curve fitting.

\section{$5 \quad$ Architecture of the control system}

The control system can be realized in three steps as Figure 5 shows.

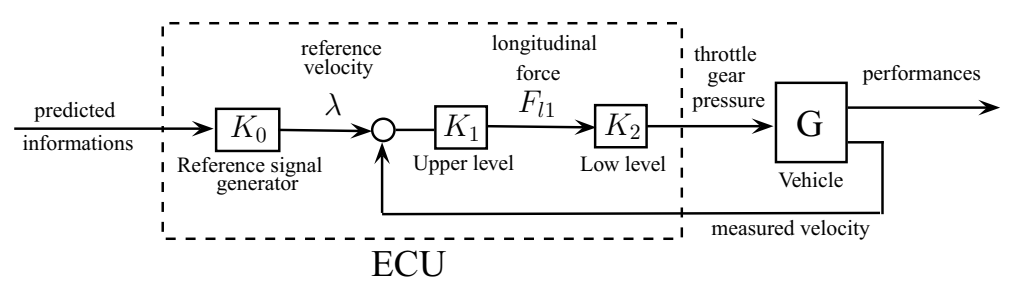

Figure 5: Implementation of the controlled system

Step 1: The aim of the first step is the computation of the reference velocity, see equation (9). The results of this computation are the weights and the modified velocity which must be tracked by the vehicle.

Step 2: In the second step the longitudinal control force of the vehicle $\left(F_{l 1}\right)$ is designed. The role of the high-level controller is to calculate this required longitudinal force.

Step 3: In third step the real physical inputs of the system, such as the throttle, the gear position and the brake pressure are generated by the low-level controller.

In the proposed method the steps are separated from each other. The reference velocity signal generator can be added to the upper-lower Adaptive Cruise Control (ACC) system. It is possible to design a reference signal generator unit almost individually, and to attach it to the ACC system. Thus the reference signal unit can be designed and produced independently from automobile suppliers, only a few vehicle data are needed. The independent implementation possibility is an important advantage in practice. The high-level controller calculates positive and negative forces as well, therefore the driving and braking systems are also actuated. Figure 6 shows the architecture of the low-level controller.

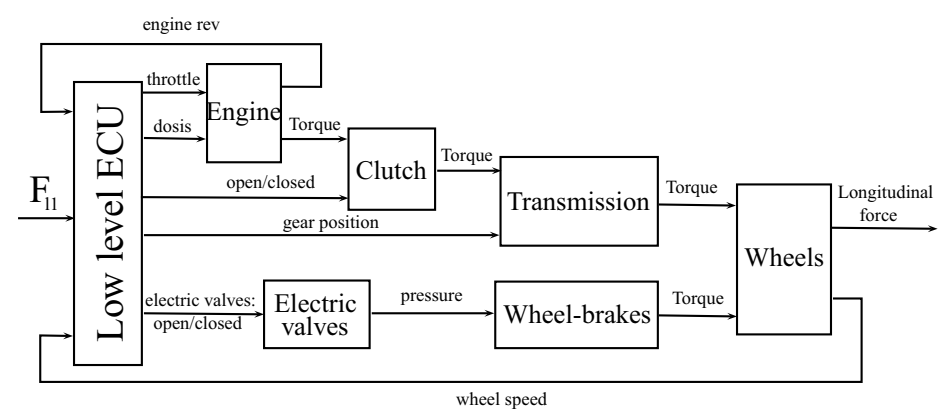

Figure 6: Architecture of the low-level controller

The schematic structure of the system contains the next parts: 
- The engine is controlled by the throttle, which could be a butterfly gate or a quantity of injected fuel. The engine-management system and the fuel-injection system have their own controllers, thus in the realization of the low-level controller only the torquerev-load characteristics of the engine are necessary. In this case the rev of the engine is measured, the required torque is computed from the longitudinal force of the highlevel controller, thus the throttle is determined by an interpolation step using a look-up table.

- The position of the automatic transmission is determined by logic functions, thus it depends on the fuel consumption and the maximum rev of the engine.

- The pressures on the cylinders of the wheels increase during braking. At normal traveling the ABS actuation is not necessary, in case of an emergency the optimal driving is overwritten by the safety requirements. The necessary braking pressure for the required braking force is computed from the ratios of the hydraulic/pneumatic parts.

\section{Simulation results}

The purpose of the simulation examples is to illustrate the operation of the proposed method and present detailed analysis of the effects of the various parameters on the method. Finally, a 56- $\mathrm{km}$-long highway with real data is analyzed.

In the examples the controller in which the velocity profile is calculated by taking the road inclinations and speed limits into consideration (Controller 1) is compared to the controller in which the reference velocity is selected constant and the road information is not considered, i.e., it is a a conventional Adaptive Cruise Control (Controller 2).

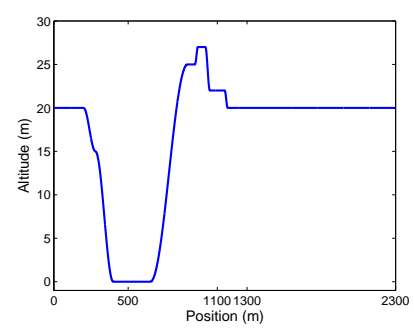

(a) Road altitude

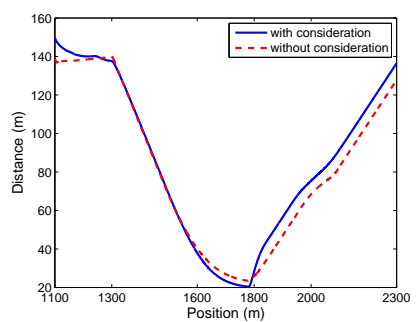

(d) Distance between vehicles

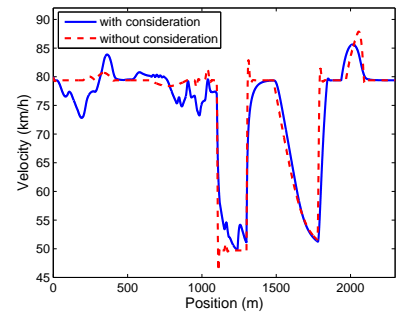

(b) Velocity of vehicle

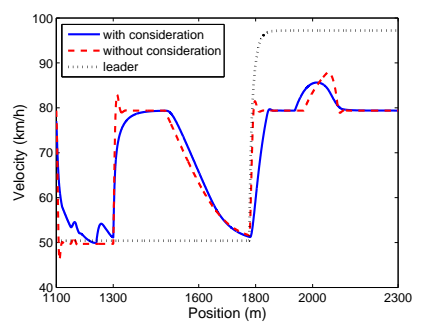

(e) Velocity of preceding vehicle

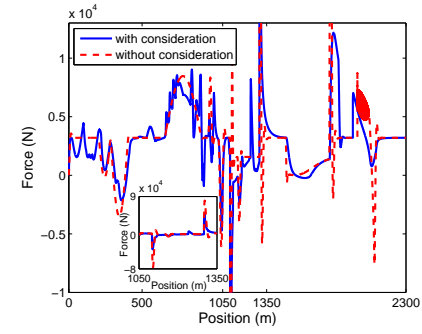

(c) Actuated force of vehicle

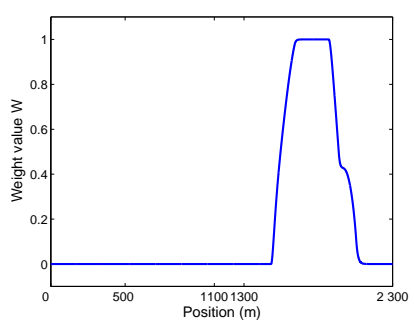

(f) Value of $W$

Figure 7: Cruise control systems on hilly road

Figure $7(\mathrm{a})$ shows the coordinates of the undulating road. It contains several uphill and downhill sections, therefore it is suitable for the analysis of the proposed method. The reference velocity is usually $v_{r e f}=80 \mathrm{~km} / \mathrm{h}$, but there is a speed limit on the road, i.e., between $1100-1300 \mathrm{~m}$ the reference velocity is $v_{\text {ref }}=50 \mathrm{~km} / \mathrm{h}$. Figure $7(\mathrm{~b})$ shows the velocity of the vehicle. The dashed line illustrates that Controller 2 tracks the predefined 
constant velocities with an acceptable error and at the downhill and uphill sections the tracking error slightly increases due to the effect of road inclinations. At the speed limit signs the velocity of the vehicle decreases and increases rapidly. At the same time the velocity in the front of the downhill section is decreased by Controller 1, since the controller reduces the required longitudinal force, see Figure $7(\mathrm{c})$. It saves energy because the decelerating effects of road disturbances are exploited when the road conditions are considered. The downhill section has an accelerating effect, therefore the velocity loss at the beginning of the downhill section can be compensated for. In the case of Controller 2 the braking system is also used to prevent the velocity increasing, while in the proposed method less braking operation is needed. In this case Controller 1 considers that after the downhill section there is a flat road section and the deceleration effect of the road disturbances can compensate for the increase of velocity. At the downhill section the consideration of the inclinations can not be exploited by the conventional method, because the disturbances do not reduce the longitudinal force. In this section Controller 2 also tracks the constant velocity. By considering the road conditions the vehicle accelerates before the uphill section, therefore it can reach a velocity at which the vehicle can travel along the uphill section with less longitudinal force. Figure 7 (c) shows that Controller 1 requires less longitudinal force, its maximal values are approximately half of the forces of Controller 2. At point $1100 \mathrm{~m}$ the velocity of the vehicle is decreased before the speed limit sign and the velocity changes.

The simulation example also shows the effect of a preceding vehicle. The preceding vehicle travels only at $50 \mathrm{~km} / \mathrm{h}$ instead of $80 \mathrm{~km} / \mathrm{h}$. Consequently, the distance between the vehicles decreases, see Figure $7(\mathrm{~d})$. Later the preceding vehicle accelerates and exceeds 80 $\mathrm{km} / \mathrm{h}$, see Figure $7(\mathrm{e})$. This simulation shows that the proposed cruise control method is able to adapt to the preceding vehicle, because the tracking vehicle approaches the preceding vehicle taking the braking distance into consideration, while in the second part the tracking vehicle avoids exceeding the speed limit and it falls behind. This solution requires radar information which is available in a conventional ACC vehicle. This velocity control is achieved by using the value of $W$ as it is shown in Figure $7(\mathrm{f})$. The simulation example shows that the controller, which takes into consideration road inclination and speed limits, requires $14 \%$ less control energy than the conventional controller and the maximum difference from the original velocity is about $7 \mathrm{~km} / \mathrm{h}$. Besides, the designed control system is able adapt to external circumstances.

The second simulation example illustrates the effects of the various parameters on the proposed method. The effects of the performance weights, the number of sampling points and the vehicle mass are analyzed. In this simulation the vehicles travel on the same road, terrain characteristics of which are illustrated in Figure 8. Figure 9 shows how the per-

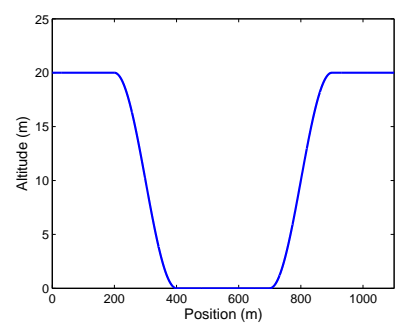

Figure 8: Shape of the analyzed road section

formance weights $R_{1}$ and $R_{2}$ influence the optimal velocity and the required longitudinal force. Different performance weights for the ratio $R_{1}$ and $R_{2}$ are analyzed. Figures show the operation of the proposed controller together with the operation of Controller 2. Since the performance weight $R_{1}$ focuses on the longitudinal force it improves results by reducing force/energy requirements. At the same time, however, the controlled system pays less attention to the difference between the momentary velocity. Similarly, if $R_{2}$ increases the 
difference between the reference velocities is less and the simulation results are closer to the results of Controller 2.

It is important to analyze the force/energy saving during the journey, see Figure 9 (a). There are sections where energy saving is very significant, e.g. more than $30 \%$. Energy savings in the downhill, uphill and flat sections are different. In the downhill section the vehicle has more possibility in the reduction of force/energy since here the longitudinal disturbances also decelerate the vehicle and there is less actuator requirement. In the uphill section there is less possibility for the control system, since there are no additional forces which help the longitudinal actuator. The result shows that the driver is able to influence the

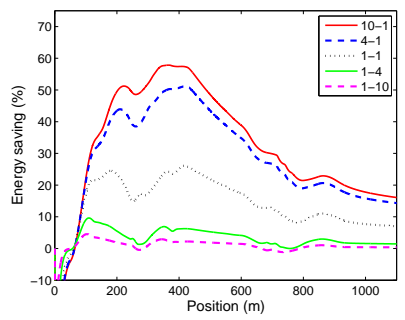

(a) Energy saving

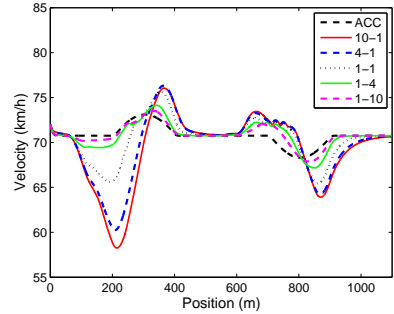

(b) Velocity of vehicle

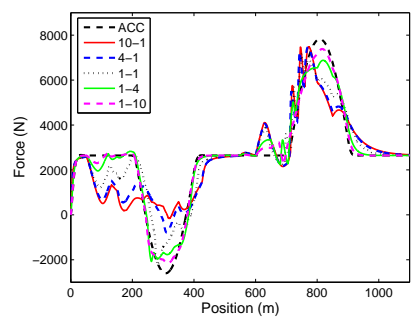

(c) Actuated force of vehicle

Figure 9: Analysis of the performance weights based on different weighting strategies

different performances by tuning the performance weights and creating a balance between saving force/energy and traveling time.

In the analysis of the control system different numbers of sampling points are selected, namely 3, 4 and 5 equidistant points on a $150 \mathrm{~m}$ road. Figure 10 shows that this parameter influences the control system significantly. The number of sampling points determines the sampling length. When the sampling length is greater the vehicle is not able to take into

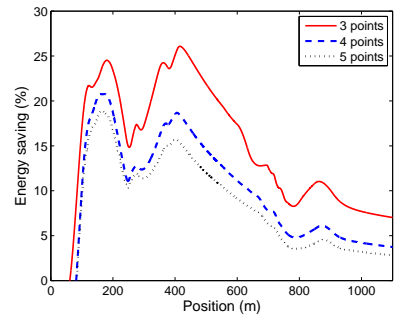

(a) Energy saving

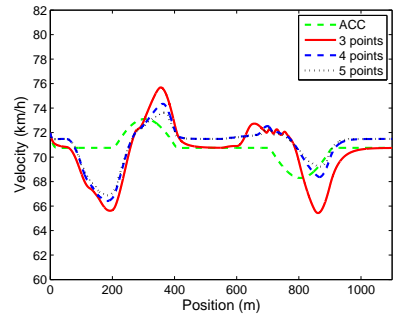

(b) Velocity of vehicle

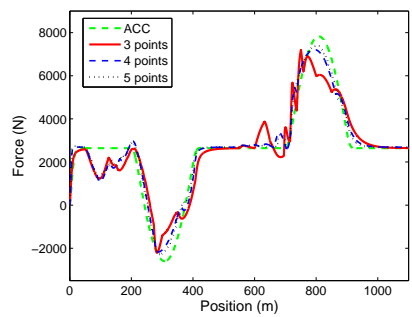

(c) Actuated force of vehicle

Figure 10: Analysis of dependence of number of sampling points

consideration the effect of inclinations, since in the control design constant vehicle dynamics is assumed. Figure 10(b) shows that the velocity decreases significantly at the beginning of the downhill section and increases significantly at the uphill section if greater sampling length is selected. The results show that fewer sampling points result in saving more force/energy. It can also be seen that by increasing the sampling length the simulation results differ more significantly from Controller 2. The analysis shows that there are relationships between the road conditions and sampling points (sampling length). For example in the case of flat roads it is enough to use fewer section points and greater sampling lengths because the slopes of the sections do not change abruptly. In the case of undulating roads it is necessary to use more section points and smaller sampling length.

Figure 11 shows the mass dependence of velocity and longitudinal force. Four vehicle masses are analyzed: $1000 \mathrm{~kg}, 3500 \mathrm{~kg}, 8000 \mathrm{~kg}$ and $12000 \mathrm{~kg}$. The other parameters are 
not changed during the simulations. Figure 11(a) shows the energy saving along the road section, which means that approximately $6-7 \%$ of energy can be saved by considering road conditions. The amount of energy saved increases slightly with the increase in the mass of the vehicle. Figure 11(b) shows that an increase in the vehicle mass makes velocity less

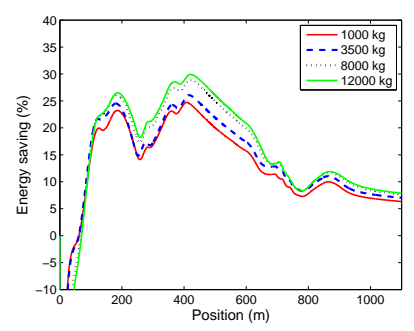

(a) Energy saving

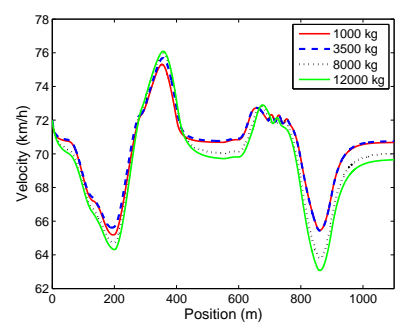

(b) Velocity of vehicle

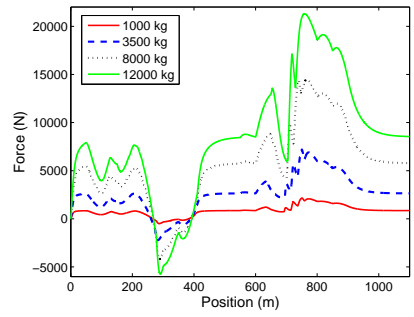

(c) Actuated force of vehicle

Figure 11: Analysis of dependence of the mass

abrupt. The computed longitudinal control forces of the vehicles are illustrated in Figure 11(c). It can be stated, that increase in the vehicle mass improves the efficiency of the presented method, which means that at fixed performance weights $R_{1}, R_{2}$, the solution of optimization (see Section 3) is closer to the solution of performance criterion Optimization 1. It can be set back with the change of $R_{1}, R_{2}$ weights.

In the last simulation a transportational route with real data is analyzed. The terrain characteristics and geographical information are those of the M1 Hungarian highway between Tatabánya and Budapest in a 56- $\mathrm{km}$-long section. In the simulation a typical F-Class truck travels along the $56 \mathrm{~km}$ route. The mass of the 6 -gear truck is $2023 \mathrm{~kg}$ and its engine power is $300 \mathrm{~kW}(402 \mathrm{hp})$. The regulated maximal velocity is $130 \mathrm{~km} / \mathrm{h}$, but the road section contains other speed limits (e.g. $80 \mathrm{~km} / \mathrm{h}$ or $100 \mathrm{~km} / \mathrm{h}$ ), and the road section also contains hilly parts. Thus, it is an acceptable route for the analysis of road conditions, i.e., inclinations and speed limits. Publicly accessible up-to-date geographical/navigational databases and visualisation programs, such as Google Earth and Google Maps, are used for the experiment.

Figure 12(a) shows the altitude of the road along the way. It shows that the chosen motorway contains several uphill and downhill sections. In this example two different controllers are compared. The first is the proposed controller, which considers the road conditions such as inclinations and speed limits and is illustrated by solid line in the figures, while the second controller is a conventional ACC system, which ignores this information and is illustrated by dashed line. Figure 12(b) shows the velocity of the vehicle with speed limits in both cases. The conventional ACC system tracks the predefined velocity speed limits as accurately as possible and the tracking error is minimal. In the proposed method the velocity of the vehicle is determined by the speed limits and simultaneously it takes the road inclinations into consideration according to the optimal requirement. In the sections of road inclinations the average relative difference between the actual velocity and the speed limit is $8 \%$ in the proposed method. Figure 12(c) shows the required longitudinal force. The high-precision tracking of the predefined velocities in the conventional ACC system often requires extremely high forces with abrupt changes in the signals. Since the proposed method uses the road inclinations and speed limits in advance in the optimization method the truck is able to travel along the road with smaller actuation. The actual physical control inputs of the brake cylinder pressure and the throttle are illustrated in Figure 12(d) and Figure $12(\mathrm{f})$, respectively. In the conventional ACC system the revolution of the engine is within smaller bounds than in the proposed controller as Figure 12(e) shows.

As a result of the road conditions less energy is required during the journey in the proposed control method, see Figures $12(\mathrm{~g})$ and 12(h). The proposed method requires smaller energy $(47.2 \mathrm{MJ})$ than the conventional method $(55.6 \mathrm{MJ})$, and the energy saving is $8.4 \mathrm{MJ}$, which is $15.1 \%$. Approximately half of this value is realized by the driveline system 


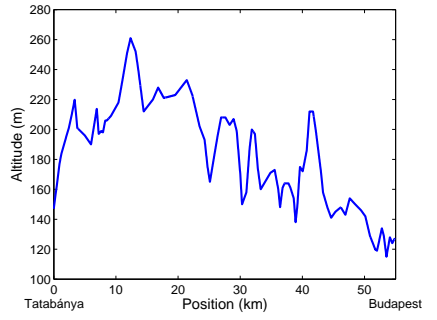

(a) Road altitude

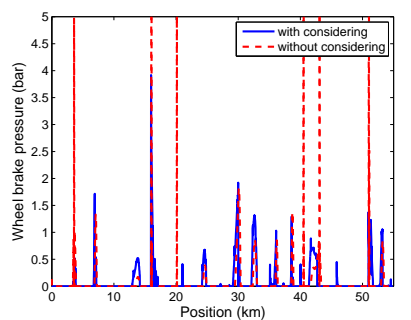

(d) Brake pressure on front wheels

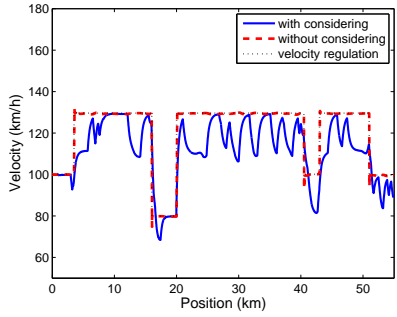

(b) Velocity of vehicle

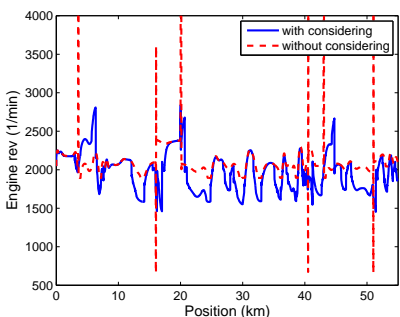

(e) Engine rev

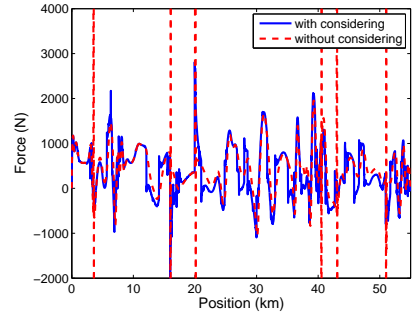

(c) Actuated force of vehicle

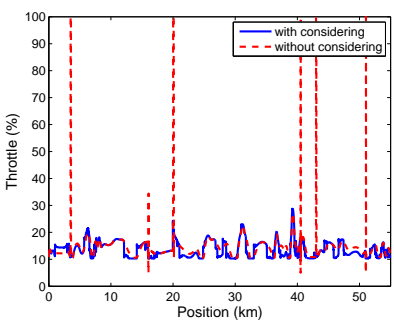

(f) Throttle

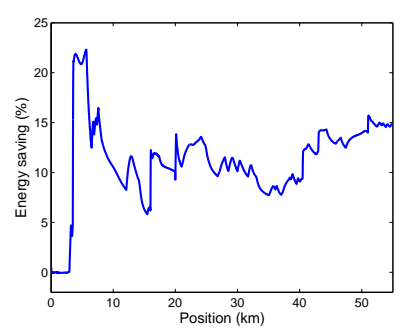

(g) Energy saving

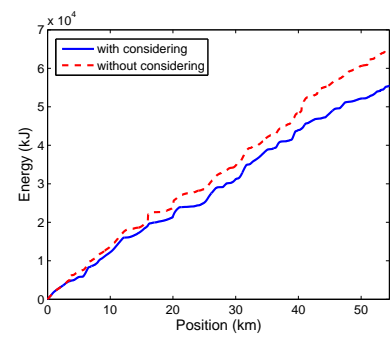

(h) Total energy

Figure 12: Real data motorway simulation

(51.4\%). The fuel consumption can also be calculated by using the following equation: $V=\left(F_{l 1} \dot{\xi}_{0}\right) /\left(\eta L_{h} \rho_{\text {fuel }}\right)$ where $\eta=0.25$ is the efficiency of the driveline system, $L_{h}=47.3$ $M J / k g$ is the heat of combustion and $\rho_{\text {fuel }}=730 \mathrm{~kg} / \mathrm{m}^{3}$ is the density of petrol. The fuel consumption of the conventional system is $6.44 l$ while that of the proposed method is 5.47 $l$, which results in $0.97 l$ reduction in fuel consumption in the analyzed $56 \mathrm{~km}$ length section. Since in this method the velocity of the vehicle may be below the permitted maximum for the given section and accelerations/decelerations are carried out more slowly and gradually than in the conventional method the duration of the journey is expected to be longer. However, the difference in the duration is only 2 minutes.

\section{Conclusion}

The paper has proposed the design of a new adaptive cruise control system, in which the longitudinal control incorporates the brake and traction forces in order to achieve the designed velocity profile. By choosing the appropriate velocity according to the road and traffic information, the number of unnecessary accelerations and brakings and their durations can be significantly reduced. The operation of the cruise control is demonstrated on a real transportation route in Hungary. Owing to the integration of vehicle actuators and road conditions the simulation results show that the designed control reduces the energy required by the actuators and it slightly increases the traveling time. An important advantage of the method is that the procedure can be implemented in an Electronic Control Unit (ECU) in 
the practice of the vehicle industry.

\section{Acknowledgment}

The research has been supported by the Hungarian National Scientific Research Fund (OTKA) through grant No. CNK-78168 which is gratefully acknowledged. The research was supported by the National Office for Research and Technology through the project "Innovation of distributed driver assistance systems for a commercial vehicles platform" (TECH_08_2/2-2008-0088).

\section{References}

[1] H. S. Bae, J. Ruy, and J. Gerdes. Road grade and vehicle parameter estimation for longitudinal control using GPS. 4th IEEE Conference on Intelligent Transportation Systems, Oakland, California, 1-6, 2001.

[2] J. Bokor and G. Balas. Linear parameter varying systems: A geometric theory and applications. 16th IFAC World Congress, Prague, 1:1-11, 2005.

[3] W. F. Faris, H. A. Rakha, R. I. Kafafy, M. Idres, and S. Elmoselhy. Vehicle fuel consumption and emission modelling: an in-depth literature review. International Journal of Vehicle Systems Modelling and Testing, 6(3):318-395, 2011.

[4] J.O. Hahn, R. Rajamani, S.H. You, and K.I. Lee. Real-time identification of roadbank angle using differential GPS. IEEE Transactions on Control Systems Technology, 12:589-599, 2004.

[5] E. Hellström, J. Åslund, and L. Nielsen. Horizon length and fuel equivalents for fueloptimal look-ahead control. Advances in Automotive Control, Munich, 1-6, 2010.

[6] E. Hellström, M. Ivarsson, J. Åslund, and L. Nielsen. Look-ahead control for heavy trucks to minimize trip time and fuel consumption. Control Engineering Practice, $17(2): 245-254,2009$.

[7] M. Ivarsson, J. Åslund, and L. Nielsen. Look ahead control - consequences of a nonlinear fuel map on truck fuel consumption. Proceedings of the Institution of Mechanical Engineers, Part D, Journal of Automobile Engineering, 223:1223-1238, 2009.

[8] U. Kiencke and L. Nielsen. Automotive control systems for engine, driveline and vehicle. Springer, 2000.

[9] I.V. Kolmanovsky and D.P. Filev. Stochastic optimal control of systems with soft constraints and opportunities for automotive applications. IEEE Conference on Control Applications, St. Petersburg, Russia, 1265-1270, 2009.

[10] I.V. Kolmanovsky and D.P. Filev. Terrain and traffic optimized vehicle speed control. IFAC Advances in Automotive Control Conference, Munich, Germany, 1-6, 2010.

[11] R. Labayrade, D. Aubert, and J.P. Tarel. Real time obstacle detection in stereovision on non flat road geometry through "v-disparity" representation. Intelligent Vehicle Symposium IEEE, 2:646-651, 2002.

[12] P. Lingman and B. Schmidtbauer. Road slope and vehicle mass estimation using Kalman filtering. Vehicle System Dynamics Supplement, 37:12-23, 2002.

[13] L. Nouveliere, M. Braci, L. Menhour, and H.T. Luu. Fuel consumption optimization for a city bus. UKACC Control Conference, Manchester, UK, 1-6, 2008. 
[14] B. Németh and P. Gáspár. Considering predicted road conditions in vehicle control design using $\mathcal{H}_{\infty}$ method. IFAC Advances in Automotive Control Conference, Munich, Germany, 1-6, 2010.

[15] B. Németh and P. Gáspár. Road inclinations in the design of lpv-based adaptive cruise control. IFAC World Congress, Milan, Italy, 1-6, 2011.

[16] H. B. Pacejka. Tyre and vehicle dynamics. Elsevier Butterworth-Heinemann, Oxford, 2004.

[17] A. Packard and G. Balas. Theory and application of linear parameter varying control techniques. American Control Conference, Workshop I, Albuquerque, New Mexico, 1997.

[18] B. Passenberg, P. Kock, and O. Stursberg. Combined time and fuel optimal driving of trucks based on a hybrid model. European Control Conference, Budapest, 1-6, 2009.

[19] H. Rakha, I. El-Shawarby, M. Arafeh, and F. Dion. Estimating path travel-time reliability. IEEE Intelligent Transportation Systems Conference, Toronto, Canada, 236-241, 2006.

[20] H. Rakha, M. Van Aerde, E.R. Case, and A. Ugge. Evaluating the benefits and interactions of route guidance and traffic control strategies using simulation. In Vehicle Navigation and Information Systems Conference, 1989. Conference Record, 296 -303, 1989.

[21] P. Sahlholm and K.H. Johansson. Road grade estimation for look-ahead vehicle control using multiple measurement runs. Control Engineering Practice, 1328-1341, 2010.

[22] D. Swaroop and J.K. Hedrick. String stability of interconnected systems. IEEE Transactions on Automatic Control, 41:349-357, 1996.

[23] A. Trachtler. Integrated vehicle dynamics control using active brake, steering and suspension systems. International Journal of Vehicle Design, 36:1-12, 2004.

[24] F. Wu, X. H. Yang, A. Packard, and G. Becker. Induced $l^{2}$-norm control for LPV systems with bounded parameter variation rates. International Journal of Nonlinear and Robust Control, 6:983-998, 1996. 